\title{
Measurement Memo I: Updated Practices in Psychological Measurement for Sexual Scientists
}

\author{
John K. Sakaluk \\ University of Victoria
}

\author{
Alexandra N. Fisher \\ University of Victoria
}

\begin{abstract}
The validity of psychological measurement is a crucial auxiliary theory underlying many sexual science studies. Although many sexuality researchers are familiar with certain elements of psychological measurement, the field of psychological measurement is a developing and evolving literature, with concepts, applications, and techniques that do not always trickle down quickly into interdisciplinary fields like sexual science. The purpose of this Measurement Memo, therefore, is to connect sexual scientists to measurement-related issues, explanations, and resources that they may not otherwise encounter in their scholarly reading. Our review focuses on those carrying out psychological measurement using theories and methods of latent variable modeling, and we identify and summarize key ideas and references that serve as good launching points for sexual scientists to begin to improve their psychological measurement practices, for beginners and seasoned users alike.
\end{abstract}

Keywords: exploratory factor analysis; confirmatory factor analysis; latent variables; measurement; taxometrics

Sexual scientists often find themselves in the position of studying intrapsychic psychological constructs, such as attitudes, attributions, beliefs, motives, perceptions, and values. Therefore, irrespective of whether one identifies as a psychologist or as hailing from some other scientific discipline(s), much of sexual science tacitly relies upon theories, methods, and applications of psychological measurement. And there is simply no escaping that measurement needs to be done well in order for subsequent tests of substantive theories to be meaningful (Meehl, 1990a, 1990b; Sakaluk, 2019).

Getting psychological measurement "right" is no small feat these days. Theoretical stances underpinning psychological measurement, for example, are complicated and often misunderstood in their implications for practice by applied users (Borsboom, 2005; Borsboom, Mellenbergh, \& van Heerden, 2003). And whereas statistical software defaults previously offered researchers a defensible escape from articulating their rationales and/or considering the impact for their analytic choices (e.g., Kaiser, 1970), the applied researcher must now be familiar with an increasing number of analytic details (e.g., Fried \& Flake, 2018; Sakaluk \& Short, 2017). Complicating matters further, the subfield of basic science in psychological measurement modeling is a living, breathing area of scholarship. New and improved techniques are continuously developed and

Correspondence concerning this paper should be addressed to John Sakaluk (sakaluk@uvic.ca; @JohnSakaluk).

Alexandra Fisher is also on Twitter (@alexfisherrr disseminated to provide researchers with increasingly sophisticated and rigorous tools for extracting psychological entities inside a person's head and representing them in a valid numeric form that a researcher can use (e.g., Jorgensen, Kite, Chen, \& Short, 2018). Interdisciplinary and multidisciplinary fields that do not occasionally check in to survey these new methodological developments are therefore at risk of being on the outside looking in for best practices in psychological measurement. And when measurement goes "wrong"--as it can, and often does (see Hussey \& Hughes, 2018; Flake \& Fried, 2019)--it can obscure important effects, produce spurious findings, or render certain comparisons entirely illogical.

As members of interdisciplinary field relying to such a large extent on psychological measurement, it is crucial for sexual scientists to remain informed about new developments and best practices in this area of applied statistics--both to ensure the quality of their own quantitative science, as well as to enable them to competently appraise the quality of others'. The purpose of our Measurement Memo is to therefore serve as a piece of knowledge brokering, in an effort to connect sexual scientists to measurement-related issues, explanations, and resources that they may not otherwise encounter in their scholarly reading. That is, our goal is not to provide exhaustive tutorials on any one (or several) technique(s) or development(s), but rather, to identify and summarize key ideas that would serve as good launching points for sexual scientists to begin to improve their psychological measurement practices. Our review focuses, in particular, on developments in the area of latent variable 
measurement and modeling (see Borsboom, 2005), as this remains the dominant approach to psychological measurement in psychology, sexuality research, and beyond.

\section{Statistical Existence Fallacy}

Sexual scientists wishing to evince the existence of an unobserved psychological entity - a latent variable (e.g., Borsboom, 2005)-will typically do one or more of the following (Flake, Pek, \& Hehman, 2017; Maul, 2017): (1) calculate a reliability estimate (usually Cronbach's alpha) for a set of items/indicators designed to reflect a latent variable, (2) use latent variable modeling such as Exploratory Factor Analysis (EFA) to evaluate the underlying structure of said variable and/or (3) examine the correlations between the new measure and pre-existing similar or theoretically related measures. After completing any one of these steps with relative success (e.g., Cronbach's alpha >.70; Flake et al., 2017; Furr \& Bacharach, 2014), sexual scientists may be tempted to conclude that a given latent variable exists and that their measure accurately reflects the latent construct (e.g., has evidence of construct validity; Furr \& Bacharach, 2014). This conclusion likely stems from the assumption that a latent variable and the items used to measure it are one of the same (Borsboom, 2006; Fried, 2017). Not only does this assumption give these statistical techniques more credit than they deserve, but it allows sexual scientists to side-step considerations of measurement quality, which, in turn, can compromise the veracity of the conclusions they draw as independent scientists and collectively as a field (Flake et al., 2017).

These statistical techniques depend upon the correlations among a set of items or variables (Rhemtulla et al., 2017). However, they cannot inuit the reasons why a selection of items or variables may be related. For example, Maul (2017) demonstrated that a set of nonsense survey items (e.g., 'To be honest, you can't really change how gavagai you are') designed to assess a similarly nonsensical latent variable 'gavagai' could produce a remarkably high reliability estimate (Cronbach's alpha $=.91 ;$ p. 54), a two-factor structure composed of high loadings (as demonstrated through EFA), and small but appreciable correlations with established self-report measures of personality (e.g., agreeableness and openness, both $r s=.09, p s<.05$; $\mathrm{p}$. 55). How could this be?

There are many possible reasons why a set of items or measures may be correlated (aside from their hypothetical linkage to a latent variable). For instance, variables measured using a similar method (e.g., self- report survey) as in Maul's (2017) study are more likely to correlate with one another due to their shared method compared to variables measured using disparate methods (e.g., self report and behavioral; Furr \& Bacharach, 2014). For this reason, a strong correlation between theoretically-related but disparately measured variables (e.g., self-reported sexual desire and recorded visits to pornography websites) might lend greater confidence in the actual existence and practical significance of a latent variable (e.g., sexual desire; Campbell \& Fiske, 1959; Cronbach \& Meehl, 1955).

Similarly, Cronbach's alpha is often misinterpreted to be a reflection of the underlying dimensionality of a latent variable whereas, in actuality, it reflects the conceptual homogeneity of a set of measurement items (Cronbach \& Shavelson, 2004). Consequently, a scale composed of conceptually and structurally similar or interchangeable items will typically produce a higher Cronbach's alpha than a scale composed of relatively dissimilar or distinct items. In Maul's (2017) case, the "gavagai" measure included both conceptually and structurally similar items (e.g., 'You have a certain amount of gavagai, and you can't really do much to change it', 'Your gavagai is something about you that you can't change very much' p. 55), which likely inflated both Cronbach's alpha and the factor loadings he obtained using EFA.

Lastly, even though Maul's (2017) participants were responding to a set of nonsensical items, the psychological processes driving their responses may not have been. It's entirely possible that participants may have projected their own meaning onto the word 'gavagai' and responded accordingly. Thus, even though Maul may have thought he was measuring 'nothing' he may still have been measuring 'something' (Rhemtulla et al., 2017). Just not the 'something' he was after. Indeed, the reasons for correlations among items or variables may not always be of particular theoretical importance or interest (Rhemtulla et al., 2017). Nonetheless, they are of substantive practical importance to sexual scientists to the extent that they can (and often do unbeknownst to us) obscure our understanding of the latent variables we are actually interested in.

In sum, these statistical techniques cannot tell us whether a latent variable actually exists, and, if it does exist, whether our measures accurately reflect its structure or breadth (Cattel, 1978). This is where we, as scientists, come in. Sexual scientists can draw on theory, common sense, and some of the techniques we discuss in this paper, in order to think critically about the form and function(s) of latent variables independently from the methods we use to measure them (Borsboom, 2006; Maul, 2017). If we believe in 
the existence of a latent variable, then it behooves us to question it, to test it, and to measure it accurately. By doing so, we can transform our assumptions about the existence of latent variables into hypotheses to corroborated or falsified thereby improving the veracity and impact of our science.

\section{Jingle-Jangle Fallacy}

Just as performing measurement modeling techniques on a set of indicators is not substantive proof of a given latent variable's existence, assigning a given latent variable a particular construct label (e.g., "attitude", "belief", "self-esteem") does not establish it as being similar to or different from another construct. Nonetheless erroneous conflations or distinctions between competing measures are common--a phenomenon described as the jingle-jangle fallacies (Marsh, 1994)--with the jingle fallacy referring to when researchers treat measures with similar names as measuring the same construct (Thorndike, 1904), and the jangle fallacy referring to when researchers treat measures with different names as measuring a different construct (Kelley, 1927).

In the context of sexual science, the jingle fallacy seems more of a risk when researchers are developing a new measure in a particular content domain and assign a particular generalist construct label (e.g., attitudes towards__ without any critical interrogation of what the intrapsychic construct would require in item format. Attitudes, for example, definitionally require valanced evaluation (; e.g., "

is wonderful."; Zanna, Kiesler, \& Pilkonis, 1970); beliefs demand cognition ( ; e.g., "I think __ is overrated."; Anderson, Lepper, \& Ross, 1980); motives capture a priori needs or wants (e.g., "I want

because I feel turned-on"; Baldwin \& Baldwin, 1986) while attributions involve post hoc meaning-making (e.g., "I wanted __ because I felt turned-on."; Heider \& Simmel, 1944); and self-esteem capture overall assessments of the self (e.g., Leary \& Baumeister, 1995), while self-efficacy is conceptualized as capturing the extent to which the self is perceived as agentic and capable (e.g., Bandura, 1982). Yet in sexual science, these construct labels are utilized in an almost casual fashion; attitudes, beliefs, and values are often considered one-in-the same, and the temporal distinction between motivation and attribution artificial and overrated. Measures may be created in which items tapping into any of these construct types may be conflated; alternatively results might diverge across studies using measures of ostensibly the same construct(s), and a great hullabaloo might be made of what could explain the difference when, in fact, it was simply that different studies were assessing different things. The net result of either possibility is that jingle fallacies get in the way of scientists pinpointing what exact processes are responsible for driving their effects of interest, a problem that can obscure both basic theory development as well as the success of applied interventions.

The jangle fallacy, meanwhile, presents a problem of a different sort. Like the jingle fallacy, errantly declaring a measure as different from another when the underlying construct is the same can obscure cumulative science by creating artificial nuance and uncertainty around which concept(s) are and are not involved or responsible for a particular empirical finding. The jangle fallacy also presents a long-term attentional concern for measure selection in sexual science: if, unknowingly, multiple measures of the same construct will permeate the published literature, then increasing burden will fall on sexual scientists to distinguish between these competing measures and articulate their decisions for selecting the one they chose--even when no substantive reason may exist. Careful attention is therefore needed by researchers, reviewers, and editors, in order to prevent the formation of a cottage-industry of specious measurement, in which artificial distinctions are drawn between otherwise conceptually exchangeable measures.

While entirely avoiding both the jingle and jangle fallacies is likely an unreasonable goal, sexual scientists should aspire to manage their collective impact. Doing so, for better and/or worse, will require researchers to stay closely attuned to developments in measurement within their subfields of interest, and to be willing to examine and scruitize competing measures--including their own--at the item content level, instead of relying on measure titles and/or summaries of measure content.

\section{Latent Dimensions vs. Latent Categories}

Beliefs about the structure of a latent construct (i.e., whether it is continuous or categorical) tend to reflect the scaling of measurement for its observed indicators (i.e., whether items are continuous or categorical): If a variable is measured on a continuous scale (e.g., sociosexual orientation; Simpson \& Gangestad, 1991), the underlying entity is also assumed to be dimensional (e.g., it exists along a continuum; Meehl, 1992). However, this is not always the case. Rather than presume the underlying structure of a latent construct, methodologists (Meehl et al., 1992, 1995; Ruscio \& Ruscio, 2008; Waller \& Meehl, 1998) have argued that the structure of a latent variable can, and should, be treated as an empirical question to be answered. 
Although such latent constructs are inherently unobservable, they leave traces of their categorical or dimensional structure in their observable indicators, which can be detected through taxometric analysis.

The structure of a latent variable has critical implications for a researcher's theorizing, assessment, analysis, and intervention strategies for the latent variable(s) in question (Ruscio, Haslam, \& Ruscio, 2006; Ruscio \& Ruscio, 2008; Sakaluk, 2019). Yet, despite the importance of understanding the underlying structure of latent variables, questions of latent structure are not commonly investigated by sexual scientists. Fortunately, recent developments in taxometric methods are easy to implement and can provide users with rigorous, simulation-based evidence of categorical or continuous structure (Ruscio \& Wang, 2017; see Sakaluk, 2019, for a review of best practices, a reproducible example, and analytic resources). In turn, if the evidence supports a dimensional model, sexual scientists can confidently move forward with their regular Exploratory or Confirmatory Factor (e.g., Fabrigar \& Wegener, 2011; Thurstone, 1947), Structural Equation Modeling, or Item Response Theory (IRT; de Ayala, 2009; Rasch, 1960) analyses. In contrast, if the evidence supports a categorical latent construct, McGrath and Walters (2012) recommend additional model comparison approaches such as Latent Class Analysis (LCA; Collins \& Lanza, 2009; if using categorical indicators), Latent Profile Analysis (LPA; Pastor, Barron, Miller, \& Davis, 2007; if using continuous indicators), or Mixture Modeling (McLachlan \& Peel, 2000; if a combination of both categorical and continuous) to appraise the number of latent categories.

Even with taxometric evidence on their side, some sexual scientists may have some misgivings about the implications or consequences of modeling latent categories in their substantive theory-testing work; rest assured, many of these concerns reflect either misunderstandings about what latent categories do or do not mean, or are equally-applicable indictments of latent continuous variables (Sakaluk, 2019). Thus, sexual scientists should consider routinely integrating taxometric analyses into their measurement research, in order to ensure they make evidence-based decisions regarding arguably the first of many large decisions that will impact the integrity of their auxiliary theory of measurement (Meehl, 1990a, 1990b).

\section{4. "Exploratory" vs. "Confirmatory" Measurement Modeling}

Exploratory Factor Analysis (EFA) and Confirmatory Factor Analysis (CFA) are functionally similar but philosophically different (Hair et al., 2006). EFA is largely an a-theoretical, bottom-up statistical approach in that it uses response patterns in observed variables to estimate plausible numbers of latent factors, and the degree to which observed variables are important manifestations of those latent factors. The measurement models generated by EFA are therefore indiscriminate in that EFA calculates a loading for every observed variable on every latent factor; EFA can be considered "theory-generating" and should be performed without a clear idea of the number and/or composition of factors.

In contrast, CFA is a theoretical, top-down statistical approach that requires sexual scientists to specify the number of factors expected to be reflected by a set of observed variables, as well as the relationships (or absence of relationships) between each of the observed variables and the latent factors. Accordingly, CFA measurement models contain discriminating patterns of loadings, and can be considered as supplying researchers with a means of corroborating or falsifying pre-existing theories of measurement through evaluations of model fit to observed data.

There is on occasion some confusion surrounding what is meant when a researcher invokes the term "confirmatory", specifically, to describe their measurement modeling analysis. The framework has some ambiguous elements, and so it is perhaps best to emphasize with the methodological consensus of what confirmatory is not. Confirming a measurement model does not involve a researcher re-running an exploratory measurement model (e.g., an EFA) on a separate - and certainly not the same! - sample, and using subjective intraocular assessment to appraise how superficially similar the measurement parameters are (cf. Rellini et al., 2005).

It is also not satisfactory to describe using a CFA on the same sample from which an EFA solution was generated as strictly "confirmatory." When the very same data generated the model under consideration, it should not be surprising to find that the model fits well according to the current data. Implicit, therefore, in the "confirmatory" label is the expectation that a researcher is independently replicating a particular model of measurement. Similarly, a researcher takes their measurement model farther and farther away from being strictly "confirmatory" when they continuously revise, refit, and reexamine it (e.g., guided by numerous "modification indices"). At some point, truly confirmatory modeling approaches require the researcher to place a particular theoretical bet of measurement, and to let the empirical chips fall where they may. 
To summarize, then, bona fide confirmatory measurement modeling strategies require: 1) a separate sample; and some combination of 2) the specification of a particular measurement model to be corroborated or falsified (or a finite, limited set of competing plausible models); and 3) the measurement model(s) being specified to include a not-entirely-indiscriminate pattern of observed variables loading onto latent variables.

\section{Beginning with Confirmatory Modeling Approaches}

Sexual scientists devote a great deal of time- and valuable manuscript real estate - to theorizing about the relationships between various psychological and sexual phenomena yet may default to EFA to evince the underlying latent variables in their models (e.g., Chadwick et al., 2017; Gomes et al., 2018). This preference for beginning with EFA over CFA may stem from the common belief that an EFA must precede the presentation of a CFA. This is simply not the case. There is no reason why CFA cannot initially be used for testing plausible and pre-existing theories of measurement, and only moving to EFA if early alreadyavailable theories of measurement appreciably fail to explain patterns of covariation in the observed indicators. Indeed, sexual scientists often work with latent variables for which there are very rich and well developed theories about their underlying structure; there exist, for example, well-established theories that point towards a two-factor structure of motivation based on approach and avoidance (e.g., Elliot \& Thrash, 2002), a five-factor structure of personality composed of openness, agreeableness, neuroticism, extraversion, and conscientiousness (e.g., the Big Five, Costa \& McCrae, 1992), and a three-factor structure of attitudes involving affective, behavioural, and cognitive components (e.g., Rosenberg \& Hovland, 1960). Similar theories also exist within sexuality research. For instance, sexual scientists have proposed many different theories of sexual desire: from one that is primarily physical/erotic(e.g., Spector, Carey, \& Steinberg, 1996) to one that is multifaceted (e.g., intimacy, eroticism, power, desirability, etc.; Chadwick et al., 2017), which could be compared initially without first defaulting to an entirely exploratory framework.

Beginning with CFA has several advantages. If the confirmatory model is supported by the data, sexual scientists can save time (and resources) by avoiding first engaging in exploratory measurement research, which upon completion would still require confirmation in another sample. This would be especially advantageous for sexual scientists working in fields where participants are difficult to recruit and/or where funding is limited. In contrast, if an initial confirmatory model is not supported, little is lost; sexual scientists can then pivot to an exploratory approach, that they likely would have otherwise pursued. In fact, CFA provides modification indices that could offer researchers insight into how an exploratory model might be re-specified to improve fit-perhaps all that is needed is to estimate a few parameters or relax a few constraints. In return, this strategy may yield a far more parsimonious model than what an entirely-unparsimonious EFA might afford.

The bottom line: Nowhere is it writ that one must begin with EFA. Sexual scientists need not use a new bottom-up measurement model for measures of latent variables - especially those that already have welldeveloped and empirically supported theories of measurement. Many scientists before us have devoted considerable time and resources to developing many basic (and ostensibly generalizable) theories of psychological measurement, so let us be brave enough to test them!

\section{Evaluating Fit of Exploratory Models to Observed Data}

Although we have argued for some distinctions between EFA and CFA, there is one important and underappreciated way in which exploratory and confirmatory measurement models are the same: they both can (and should) have their adequacy for explaining underlying data quantified and interpreted. Yet whereas fit indices are routinely calculated, reported, and relied upon when conducting research with confirmatory measurement models (e.g. Hu \& Bentler, 1999), rarely is this the case when conducting research with exploratory measurement models (see Fabrigar et al., 1999; Sakaluk \& Short, 2017). CFA yields a model-implied variance-covariance for modeled indicator variables (i.e., what the model predicts (co)variances should be), against which a researcher's observed variance-covariance matrix of modeled indicator variables (i.e., what the (co)variances actually were) can be compared (Little, 2013) - this is the bedrock principle underlying the calculation of all of the indices of model fit (e.g., $\mathrm{Hu} \&$ Bentler, 1999). But EFA is grounded in the same statistical model of latent variables as CFA, and as such, it yields a model-implied correlation matrix (see Fabrigar \& Wegener, 2011) that can (and should) be compared against the observed correlation matrix of modeled variables to provide similar indices.

The absence of model fit indices from exploratory work can impede a scientist's research later as one 
should not be optimistic about being able to confirm an exploratory model in a new sample when the data illustrated the original exploratory model did not fit the data well. Conducting confirmatory measurement tests when exploratory measurement model fit is, at best, unknown (and at worst, poor) is potentially throwing good research resources after bad. Incorporating fit indexes into exploratory measurement modeling approaches like EFA (see Sakaluk \& Short, 2017, for analytic resources for Mplus, R, SAS, and SPSS) can therefore help to ensure that sexual scientists wisely invest subsequent resources (e.g., recruiting time/cost for a new sample) attempting to corroborate or falsify exploratory measurement models that are, at the very least, empirically plausible.

\section{Unfounded Researcher-Bias Against Correlated Factors and Cross-Loading Indicators}

When researchers using EFA extract two or more dimensions, the must somehow select among the infinite orientations of the factors in multidimensional space. A researcher selecting a given orientation is facilitated by their rotating the factor solution, using a particular statistical criterion (e.g., varimax, quartimin) to produce a pattern of loadings that hopefully demonstrates simple structure. Although sexual scientists are aware of the need to rotate multi-factor solutions, and that simple structure is a desirable property for a given factor solution, there remain misunderstandings about what actually constitutes-and does not constitute--simple structure. As a result of these misunderstandings, sexual scientists continue to gravitate towards rotation options that will, ironically, tend to produce worse simple structure for most psychological measures (Sakaluk \& Short, 2017), and/or seek out considerably less parsimonious analytic frameworks (Asparouhov \& Muthén, 2009).

The two key misunderstandings about the makeup of factor solutions with simple structure could be summarized as follows: 1) factor solutions with uncorrelated factors are simpler than factor solutions with correlated factors; and 2) the pursuit of simple structure demands a solution in which there are no variables cross-loading onto multiple factors. As it stands, neither of these are true. Here is L.L. Thurstone-developer of the simple structure criteria--in his own words:

Rotate to a solution in which:

1. Each row of the factor matrix $\boldsymbol{V}$ should have at least one zero

2. For each column $\boldsymbol{p}$ of the factor matrix $\boldsymbol{V}$ there should be a distinct set of $r$ linearly independent tests whose factor loadings $\boldsymbol{v}_{j p}$ are zero

3. For every pair of columns of $\boldsymbol{V}$ there should be several tests who entries $v_{j p}$ vanish in one column but not the other

4. For every pair of columns of $\boldsymbol{V}$, a large proportion of the tests should have zero entries in in both columns. This applies to factor problems with four or five or more common factors

5. For every pair of columns there should preferably be only a small number of tests with non-vanishing entries in both columns (Thurstone, 1947, p. 335)

Thus, no where in the entire set of criteria is the (un)correlatedness of the factor solution mentioned and Thurstone's fifth criterion directly speaks against the need for the total absence of cross-loadings. But why do these details matter?

When conducting EFA, the misunderstanding of simple structure as demanding an uncorrelated model of latent variables often motivates researchers to mistakenly use orthogonal rotation methods (Fabrigar \& Wegener, 2011; Sakaluk \& Short, 2017), which are often also misunderstood as "making" latent variables uncorrelated. Orthogonal rotation methods, however, do not "make" the latent factors uncorrelated--they simply do not permit the estimation of factor correlations whether they be present or absent. And when factor correlations are truly present, orthogonal rotation methods will usually render solutions with worse simple structure, while an oblique rotation would yield better simple structure, with a solution containing a more interpretable pattern of discriminating loadings.

Failing to model a true cross-loading variable, meanwhile, creates downstream problems for the applied researcher; the shared variance in the should-be cross-loading item is 'pushed' into other locations of a given factor model--locations where this shared variance should not be. This modeling error is expressed in one of two ways (see Asparouhov \& Muthén, 2009), either: 1) prompting the researcher to errantly specify correlated residual terms between the item and other items from the latent variable onto which it should cross-load--quickly resulting in a needlessly complicated a model; or 2) artificially inflating the correlation between the latent variable onto which the item is specified as loading exclusively, and the latent variable for which the cross-loading has been suppressed--leading a researcher to be more likely to mistakenly infer the presence of a (larger) correlation. Conversely, a researcher might be inclined to remove all cross-loading items with cross-loadings. In doing so, however, they would be artificially 
restricting the construct space that is captured in quantitative measurement models. The affective experience of feeling "surprise", for example, can have both positive (e.g., unexpected journal acceptance) and negative (e.g., unexpected journal rejection) qualities, but that should not motivate a researcher to exclude it from being considered a legitimate indicator of either/both valances of affect (Watson, Clark, \& Tellegen, 1988), and if they did, it would be fair to view the resulting measurement model as a conceptually deprived oversimplification.

Correlated factors and selectively cross-loading indicator variables are allowed in strong measurement models of latent variables. Moreover, these features can be crucial parts of the construct space of a set of modeled latent variables; sexual scientists should anticipate them, appreciate them, and model them.

\section{Precariousness of Hu and Bentler's (1999) Cutoffs for Model Fit Indices}

Few statistical conventions-perhaps with the exception of Baron and Kenny's then-ground-breaking (and now outdated) approach to testing mediation (1986, currently cited over 82,000 times according to Google Scholar) - have achieved the level of exaltation that the cutoffs for CFA/SEM fit indices proposed by $\mathrm{Hu}$ and Bentler (1999, currently cited over 56,000 times according to Google Scholar) have enjoyed. If there were a Bible of CFA/SEM, the $\mathrm{Hu}$ and Bentler cutoffs would surely be the most widely read and beloved gospel: if thou wouldst be published, thou must have CFI/TLI $>=.95$, and RMSEA/SRMR $<.08$ (and ideally .05)! Gone unnoticed by the masses, however, is that for some time latent variable methodologists (and indeed, $\mathrm{Hu}$ and Bentler themselves) have realized the precariousness of these cutoffs for what is considered "good" model fit (e.g., Jorgensen, Kite, Chen, \& Short, 2018, for an accessible review see McNeish, An, \& Hancock, 2018). As it turns out, the same cutoffs may be too lax, quite reasonable, or too strict depending on other features of the measurement modeling context.

Before describing what features of measurement alter the (in)appropriateness of the $\mathrm{Hu}$ and Bentler (1999) cutoffs, it is worth remembering what model fit indices provide the researcher: a means of detecting when they have specified a part of their measurement (e.g., the estimation or omission of a factor loading) and structural models (e.g., the estimation or omission of a correlation between two factors) that are not supported by their observed data. The function of preventing researcher retention of bad models was at the very heart of $\mathrm{Hu}$ and Bentler's (1999) original research; they deliberately simulated instances in which models were misspecified (e.g., omitting an important latent correlation) and examined cutoffs for different fit indices that would reliably flag the problem for researchers.

Although $\mathrm{Hu}$ and Bentler (1999) varied many features across their simulations in an effort to make their findings more robust and generalizable, one feature that was held constant in there models was the standardized loadings for indicators onto the latent variables $(\lambda=.70-.80)$. And as it turns out, the typical standardized loading value in a measurement model-which can be thought as reflecting some aspect of factor reliability--is a crucial modifier of how sensitive fit indices are to substantive model misspecification. Dubbed by some as "the Reliability Paradox" (Hancock \& Mueller, 2011), the Hu and Bentler (1999) cutoffs will be too lenient (allowing researchers to retain badly misspecified models) when standardized loadings are lower than their $\lambda=.70-.80$ range, and too strict (prompting researchers to reject good models) when standardized loadings are higher than their $\lambda=$ $.70-.80$ range.

While there have been a number of simulations and commentaries on the precariousness of the $\mathrm{Hu}$ and Bentler (1999) cutoffs that align with the concerns underlying the Reliability Paradox, the review by McNeish and colleagues stands to be a game-changer by virtue of its simultaneously comprehensive coverage of the previous literature, and the broad accessibility of its practical analytic recommendations. We cannot recommend enough for those using CFA/SEM fit indices in their own research to read the paper, but in short, McNeish and colleagues use simple simulations to demonstrate just how overly-liberal or too-strict the Hu and Bentler (1999) cutoffs can be with measurement models departing from the $\lambda=.70-.80$ range of $\mathrm{Hu}$ and Bentler's original study. When $\lambda \mathrm{s}=$ .40 (a plausible level given Tabachnick and Fidell, 2012, who recommend retaining indicators with $\lambda>=$ $.35), \mathrm{Hu}$ and Bentler's (1999) cutoffs are too liberal-even stricter cutoffs of RMSEA $<.04$ and CFI $>.975$ would be insufficient for reliably detecting misspecified models. When $\lambda \mathrm{s}=.90$, meanwhile, $\mathrm{Hu}$ and Bentler's (1999) cutoffs are too strict, as cuttoffs of RMSEA $<.20$ and CFI $>.775$ would be up to the task of reliably detecting the same level of misspecification.

What is the applied CFA/SEM researcher to do, then, in light of the Reliability Paradox? Ideally, accessible tools will eventually follow to help researchers precisely calibrate their models to reasonable fit index cut-offs. Although new analytic techniques are already available to assist in this calibration (see Jorgensen et al., 2018), they likely 
require a degree of sophistication beyond what the typical everyday user is prepared to indulge. Sexual scientists therefore wishing to revise their analytic practice to take model reliability into account when evaluating model fit might consider calculating average or median standardized loadings, and using McNeish et al.'s Table 1 to derive rough cut-offs that may be more reasonable, until more accessible and comprehensive tools emerge.

\section{Keeping Substantive Analyses in Latent Space}

With our final point in this initial Measurement Memo, we offer a plea for sexual scientists to consider performing their substantive non-measurement related tests in latent variable modeling frameworks like SEM and/or mixture modeling. Too often it is the case that researchers first use latent variable analysis frameworks (e.g., EFA, CFA) to create and/or evaluate their theories of measurement--revealing complexity, nuance, and the untenability of over-simplifying assumptions--and then pivot to using simple methods of numeric representation of their measures (e.g., sum and average scores) that ignore all of the previouslyestablished nuance of measurement.

Take for example the practice of creating an average score from a set of items after having subjected them to a factor analysis. Researchers using a factor analysis in most cases will find that items load to different extents onto their latent variables, yet they will then proceed to create an unweighted average score that presumes all items are equally strong indicators of the underlying factor. The resulting analyses using the oversimplified numeric representation (e.g., a t-test using an average score) would be understandable if there were not other alternatives--or at least none that were reasonably accessible. Yet in truth, if one has already gone to the trouble of specifying a latent measurement model, there is very little additional coding labor required to bring the substantive testing (e.g., comparing groups, testing correlations, evaluating regression models) into that latent framework.

Yet another example of this kind disconnect between measurement models and substantive analyses occurs when researchers establish multiple correlated factors as underlying their observed indicators, and then create a composite for an "overall" score of some sort, ostensibly intending to stand in as proxy for a generalized factor underlying all the items at hand. A generalized factor, however, is a measurement model in search of evidence, and researchers rarely take the steps required to test the support for either a higherorder or bifactor model (e.g., Chen, Hayes, Carver, Laurenceau, \& Zhang, 2012; Morin, Arens, \& Marsh,
2016). In absence of any formal support for a generalized factor, the creation of an unweighted overall aggregate thereby not only obscures the subtleties of the relationship between indicators and latent variable(s), but also presumes an entirely different measurement model than that which was tested.

The benefits of keeping substantive analyses in latent space are many; in particular the estimated effects are typically more accurate (Ledgerwood \& Shrout, 2011), as they have had the influence of random error and other factors not-yet modeled partitioned from influencing the tests at hand (Little, 2013), and these tests can be further immunized against competing explanations that would undermine their validity (Vandenberg \& Lance, 2000). To go to the trouble, then, of assessing a nuanced theory of measurement, only to gloss over it in the course of the analyses that follow makes little sense as an analytic strategy; making fewer assumptions about their data and honouring the subtleties of their tested measurement models by using analytic frameworks that can accommodate them would therefore bring sexual scientists' values much closer into alignment with their statistical practices.

\section{Conclusions}

When quantitative psychological variables are called upon by sexual scientists to stand in their theorytesting process as determinants, intermediary processes, and/or outcomes, it is crucial that they be measured and modeled appropriately (Flake \& Fried, 2019; Fried \& Flake, 2018; Meehl, 1990a, 1990b; Sakaluk, 2019). With the rate at which the literature of psychological measurement theory and analytic techniques is developing, however, it can be difficult for an interdisciplinary field like sexual science to stay abreast of current best practices. This Measurement Memo is therefore an attempt--and certainly not the last--to reduce the divide between the methodological developers and interdisciplinary users of psychological measurement principles. Moving forward, methodological specialists in other areas of sexual science should be encouraged to produce Memos of their own, as the health of sexual science will depend on our field's ability to recognize changes in best practices, increase its capacity, and maintain its rigor in deploying the diverse methodologies that enrich our field. 


\section{References}

Abele, A. E., \& Spurk, D. (2009). The longitudinal impact of selfefficacy and career goals on objective and subjective career success. Journal of Vocational Behavior, 74(1), 53-62. https://doi.org/10/crh36x

Anderson, C. A., Lepper, M. R., \& Ross, L. (1980). Perseverance of social theories: The role of explanation in the persistence of discredited information. Journal of Personality and Social Psychology, 39, 1037-1049. https://doi.org/10.1037/h0077720

Asparouhov, T., \& Muthén, B. (2009). Exploratory structural equation modeling. Structural Equation Modeling: A Multidisciplinary Journal, 16(3), 397-438. https://doi.org/10.1080/10705510903008204

Baldwin, J.D., \& Baldwin, J.L. (1986). Behavior principles in everyday life (2nd ed.). Englewood Cliffs, NJ: Prentice Hall.

Bandura, A. (1982). Self-efficacy mechanism in human agency. American Psychologist, $\quad 37, \quad$ 122-147. https://doi.org/10.1037/0003-066X.37.2.122

Baron, R. M., \& Kenny, D. A. (1986). The moderator-mediator variable distinction in social psychological research: Conceptual, strategic, and statistical considerations. Journal of Personality and Social Psychology, 51, 1173-1182. https://doi.org/10.1037/0022-3514.51.6.1173

Baumeister, R. F., \& Leary, M. R. (1995). The need to belong: Desire for interpersonal attachments as a fundamental human motivation. Psychological Bulletin, 117, 497-529. https://doi.org/10.1037/0033-2909.117.3.497

Borsboom, D. (2005). Measuring the Mind: Conceptual Issues in Contemporary Psychometrics. Cambridge, United Kingdom: Cambridge University Press. https://doi.org/10.1017/CBO9780511490026

Borsboom, D. (2006). The attack of the psychometricians. Psychometrika, 71, 425-440. https://doi.org/10.1007/s11336$\underline{006-1447-6}$

Borsboom, D., Mellenbergh, G. J., \& van Heerden, J. (2003). The theoretical status of latent variables. Psychological Review, 110, 203-219. https://doi.org/10.1037/0033-295X.110.2.203

Campbell, D. T., \& Fiske, D. W. (1959).Convergent and discriminant validation by the multitrait, multi-method matrix. Psychological Bulletin, 56, 81-105. https://doi.org/10.1037/h0046016

Cattell, R.B. (1978) The scientific use of factor analysis in behavioral and life sciences. Plenum, New York. https://doi.org/10.1007/978-1-4684-2262-7

Chadwick, S. B., Burke, S. M., Goldey, K. L., Bell, S. N., \& van Anders, S. M. (2017). Sexual desire in sexual minority and majority women and men: The Multifaceted Sexual Desire Questionnaire. Archives of Sexual Behavior, 46, 2465-2484. https://doi.org/10.1007/s10508-016-0895-z

Chen, F. F., Hayes, A., Carver, C. S., Laurenceau, J. P., \& Zhang, Z. (2012). Modeling general and specific variance in multifaceted constructs: A comparison of the bifactor model to other approaches. Journal of Personality, 80, 219-251. https://doi.org/10.1111/j.1467-6494.2011.00739.x
Collins, L. M., \& Lanza, S. T. (2009). Latent class and latent transition analysis: With applications in the social, behavioral, and health sciences. Hoboken, NJ: Wiley. https://doi.org/10.1002/9780470567333

Costa, P.J., Jr., \& McCrae, R.R. (1992). The NEO-PI-R: Professional manual. Odessa, FL: Psychological Assessment Resources.

Cronbach, L. J., \& Meehl, P. E.(1955). Construct validity in psychological tests. Psychological Bulletin, 52, 281-302. https://doi.org/10.1037/h0040957

Cronbach, L. J., \& Shavelson, R. J. (2004). My current thoughts on coefficient alpha and successor procedures. Educational and Psychological Measurement, 64, 391-418. https://doi.org/10.1177/0013164404266386

de Ayala, R. J. (2009). The theory and practice of item response theory. New York, NY: Guilford Press.

Elliot, A. J., \& Thrash, T. M. (2002). Approach-avoidance motivation in personality: Approach and avoidance temperaments and goals. Journal of Personality and Social Psychology, 82, 804-818. https://doi.org/10.1037/00223514.82.5.804

Fabrigar, L. R., Wegener, D. T., MacCallum, R. C., \& Strahan, E. J. (1999). Evaluating the use of exploratory factor analysis in psychological research. Psychological Methods, 4, 272-299. https://doi.org/10.1037/1082-989X.4.3.272

Fabrigar, L. R., \& Wegener, D. T. (2011). Exploratory factor analysis. New York, NY: Oxford Press. https://doi.org/10.1093/acprof:osobl/9780199734177.001.0001

Flake, J.K., Pek, J., \& Hehman, E. (2017)._Construct validation in social and personality research: Current practice and recommendations. Social Psychological and Personality Science. $\quad 8, \quad 370-378$ https://doi.org/10.1177/1948550617693063

Fried, E. I. (2017). What are psychological constructs? On the nature and statistical modelling of emotions, intelligence, personality traits and mental disorders. Health Psychology Review, 11, 130-134. https://doi.org/10.1080/17437199.2017.1306718

Fried, E. I., \& Flake, J.K. (2018). Measurement matters. APS Observer. 31 (3).

Flake, J. K., \& Fried, E. I. (2019, January 17). Measurement schmeasurement: Questionable measurement practices and how to avoid them. https://doi.org/10.31234/osf.io/hs7wm

Furr, R.M., \& Bacharach, V.R. (2014). Psychometrics: An introduction. Thousand Oaks, California: Sage.

Gomes, A. L. Q., Janssen, E., Santos-Iglesias, P., Pinto-Gouveia, J., Fonseca, L. M., \& Nobre, P. J. (2018). Validation of the Sexual Inhibition and Sexual Excitation Scales (SIS/SES) in Portugal: Assessing gender differences and predictors of sexual functioning. Archives of Sexual Behavior, 47, 1721-1732. https://doi.org/10.1007/s10508-017-1137-8

Gray, J. A. (1987). The psychology of fear and stress. Cambridge, England: Cambridge University Press.

Hair, J.F., Black, W.C., Babin, B.J., Anderson, R.E., \& Tatham, R.L. (2006). Multivariate data analysis. New Jersey: Prentice Hall. 
Hancock, G. R., \& Mueller, R. O. (2011). The reliability paradox in assessing structural relations within covariance structure models. Educational and Psychological Measurement, 71, 306-324. https://doi.org/10.1177/0013164410384856

Heider, F. \& Simmel, M. (1944). An experimental study of apparent behavior. American Journal of Psychology, 57, 243-259. https://doi.org/10.2307/1416950

Hu, L., \& Bentler, P. M. (1999). Cutoff criteria for fit indexes in covariance structure analysis: Conventional criteria versus new alternatives. Structural Equation Modeling: A Multidisciplinary Journal, 6, 1-55. https://doi.org/10.1080/10705519909540118

Hussey, I., \& Hughes, S. (2018). Hidden invalidity among fifteen commonly used measures in social and personality psychology. https://doi.org/10.31234/osf.io/7rbfp

Jorgensen, T. D., Kite, B. A., Chen, P.-Y., \& Short, S. D. (2018). Permutation randomization methods for testing measurement equivalence and detecting differential item functioning in multiple-group confirmatory factor analysis. Psychological Methods, 23, 708-728. https://doi.org/10.1037/met0000152

Kaiser, H. F. (1970). A second generation little jiffy. Psychometrika, 35(4), 401-415. https://doi.org/10.1007/BF02291817

Kelley, T. L. (1927). Interpretation of educational measurements. New York, NY: World book.

Ledgerwood, A., \& Shrout, P. E. (2011). The trade-off between accuracy and precision in latent variable models of mediation processes. Journal of Personality and Social psychology, 101, 1174-88. https://doi.org/10.1037/a0024776

Little, T. D. (2013). Longitudinal structural equation modeling. New York, NY: Guilford Press.

Maul, A. (2017). Rethinking Traditional Methods of Survey Validation. Measurement: Interdisciplinary Research and Perspectives, 15 , $51-69$. https://doi.org/10.1080/15366367.2017.1348108

Marsh, H. W. (1994). Sport motivation orientations: Beware of the jingle-jangle fallacies. Journal of Sport \& Exercise Psychology, 16, 365-380. https://doi.org/10.1123/jsep.16.4.365

McGrath, R. E., \& Walters, G. D. (2012). Taxometric analysis as a general strategy for distinguishing categorical from dimensional latent structure. Psychological Methods, 17, 284-293. https://doi.org/10.1037/a0026973

McLachlan, G., \& Peel, D. (2000). Finite Mixture Models. Wiley: New York. https://doi.org/10.1002/0471721182

McNeish, D., An, J., \& Hancock, G. R. (2018). The thorny relation between measurement quality and fit index cutoffs in latent variable models. Journal of Personality Assessment, 100, 43-52. https://doi.org/10.1080/00223891.2017.1281286

Meehl, P. E. (1990a). Appraising and amending theories: The strategy of Lakatosian defense and two principles that warrant it. Psychological Inquiry, 1, 108-141. https://doi.org/10.1207/s15327965pli0102_1

Meehl, P. E. (1990b). Why summaries of research on psychological theories are often uninterpretable. Psychological Reports, 66, 195-244. https://doi.org/10.2466/pr0.1990.66.1.195

Meehl, P. E. (1992). Factors and taxa, traits and types, differences of degree and differences in kind. Journal of Personality, 60, 117-174. https://doi.org/10.1111/j.1467-6494.1992.tb00269.x
Meehl, P. E. (1995). Bootstraps taxometrics: Solving the classification problem in psychopathology. American Psychologist, 50, 266-275. https://doi.org/10.1037/0003066X.50.4.266

Morin, A.J.S., Arens, A., \& Marsh, H. (2016). A bifactor exploratory structural equation modeling framework for the identification of distinct sources of construct-relevant psychometric multidimensionality. Structural Equation Modeling, $\quad 23, \quad 116-139$ https://doi.org/10.1080/10705511.2014.961800

Rasch, G. (1960). Probablistic models for some intelligence and attainment tests.. Copenhagen, Denmark: Danmarks Paedogogiske Institut.

Pastor, D. A., Barron, K. E., Miller, B., \& Davis, S. L. (2007). A latent profile analysis of college students' achievement goal orientation. Contemporary Educational Psychology, 32, 8-47. https://doi.org/10.1016/j.cedpsych.2006.10.003

Rellini, A. H., Nappi, R. E., Vaccaro, P., Ferdeghini, F., Abbiati, I., \& Meston, C. M. (2005). Validation of the McCoy Female Sexuality Questionnaire in an Italian Sample. Archives of Sexual Behavior, 34, 641-647. https://doi.org/10.1007/s10508-0057915-8

Rhemtulla, M., Borsboom, D., \& van Bork, R. (2017). How to Measure Nothing. Measurement: Interdisciplinary Research and Perspectives, 15, 95-97. https://doi.org/10.1080/15366367.2017.1369785

Rosenberg, M. J. , \& Hovland, C. I. (1960). Cognitive, affective, and behavioral components of attitudes. In M. Rosenberg, C. Hovland, W. McGuire, R. Abelson, \& J. Brehm (Eds.), Attitude organization and change (pp. 1-14). New Haven, CT: Yale University Press.

Ruscio, J., Haslam, N., \& Ruscio, A. M. (2006). Introduction to the taxometric method: A practical guide. Mahwah, NJ, US: Lawrence Erlbaum Associates Publishers.

Ruscio, J., \& Ruscio, A. M. (2008). Categories and dimensions: Advancing psychological science through the study of latent structure. Current Directions in Psychological Science, 17, 203 207. https://doi.org/10.1111/j.1467-8721.2008.00575.x

Ruscio, J., \& Wang, S. (2017). RTaxometrics: Taxometric analysis. Retrieved from https://CRAN.Rproject.org/package $=$ RTaxometrics

Sakaluk, J. K., \& Short, S. D. (2017). A methodological review of exploratory factor analysis in sexuality research: Used practices, best practices, and data analysis resources. Journal of Sex Research, 54, 1-9. https://doi.org/10.1080/00224499.2015.1137538

Sakaluk, J.K. (2019). Expanding statistical frontiers in sexual science: Taxometric, invariance, and equivalence testing. Journal of Sex Research. Advance online publication. https://doi.org/10.1080/00224499.2019.1568377

Spector, I., Carey, M., \& Steinberg, L. (1996). The Sexual Desire Inventory: Development, factor structure, and evidence of reliability. Journal of Sex \& Marital Therapy, 22,175-190. https://doi.org/10.1080/00926239608414655

Simpson, J. A., \& Gangestad, S. W. (1991). Individual differences in sociosexuality: Evidence for convergent and discriminant validity. Journal of Personality and Social Psychology, 60, 870883. http://dx.doi.org/10.1037/0022-3514.60.6.870 
Tabachnick, B. G., \& Fidell, L. S. (2012). Using multivariate statistics (5th ed.). New York: Allyn and Bacon.

Thurstone, T. T. (1947). Multiple factor analysis. Chicago, Il: University of Chicago Press.

Thorndike, E. L. (1904). An introduction to the theory of mental and social measurements. New York: Teachers College, Columbia University. https://doi.org/10.1037/13283-000

Vandenberg, R. J., \& Lance, C. E. (2000). A review and synthesis of the measurement invariance literature: Suggestions, practices, and recommendations for organizational research. Organizational Research Methods, 3, 4-70. https://doi.org/10.1177/109442810031002
Waller, N. G., \& Meehl, P. E. (1998). Multivariate taxometric procedures: Distinguishing types from continua. Thousand Oaks, CA: Sage

Watson, D., Clark, L. A., \& Tellegen, A. (1988). Development and validation of brief measures of positive and negative affect: The PANAS Scales. Journal of Personality and Social Psychology, 54, 1063-1070. https://doi.org/10.1037/0022-3514.54.6.1063

Zanna, M.P., Kiesler, C.A., \& Pilkonis, P.A. (1970). Positive and negative attitudinal affect established by classical conditioning. Journal of Personality and Social Psychology, 14, 321-328. https://doi.org/10.1037/h0028991 